\title{
Tunable infrared asymmetric light transmission and absorption via graphene-hBN metamaterials
}

\author{
Cite as: J. Appl. Phys. 126, 193102 (2019); doi: 10.1063/1.5118887 \\ Submitted: 5 July 2019 . Accepted: 17 October 2019 . \\ Published Online: 15 November 2019

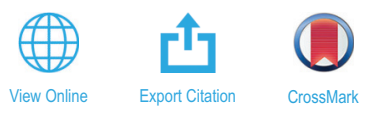

Hodjat Hajian, ${ }^{1, a)}$ (D) Amir Ghobadi, ${ }^{1,2}$ (D) Andriy E. Serebryannikov, ${ }^{3,4}$ Bayram Butun,

Guy A. E. Vandenbosch, ${ }^{4}$ (D) and Ekmel Ozbay ${ }^{1,2,5, a)}$

\author{
AFFILIATIONS \\ ${ }^{7}$ NANOTAM—Nanotechnology Research Center, Bilkent University, 06800 Ankara, Turkey \\ ${ }^{2}$ Department of Electrical and Electronics Engineering, Bilkent University, 06800 Ankara, Turkey \\ ${ }^{3}$ Faculty of Physics, Adam Mickiewicz University, 61-614 Poznan, Poland \\ ${ }^{4}$ ESAT-TELEMIC, Katholieke Universiteit Leuven, B-3000 Leuven, Belgium \\ ${ }^{5}$ Department of Physics and UNAM-Institute of Materials Science and Nanotechnology, Bilkent University, 06800 Ankara, Turkey
}

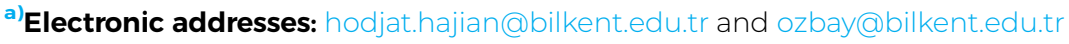

\begin{abstract}
We theoretically prove in this paper that using planar multilayer graphene-hexagonal boron nitride (hBN) metamaterials (GhMMs) can yield ultrabroadband and high-contrast asymmetric transmission (AT) and asymmetric absorption (AA) of light. The AA and AT features are obtained in the far-infrared (FIR) and mid-infrared (MIR) regions for normally incident light with transverse magnetic polarization. Here, the GhMMs are integrated with two asymmetric gratings of Ge and are composed of alternating multilayers of graphene (11 multilayers) and hBN layers (10 layers). Moreover, the total subwavelength thickness of the hybrid structures is about $3 \mu \mathrm{m}$, being less than half of the free-space wavelength up to nearly $50 \mathrm{THz}$. This approach-which is similar to the one introduced by Xu and Lezec [Nat. Commun. 5, 4141 (2014)] for a passive hyperbolic metamaterial operating in the visible range-is based on the excitation of high- $\beta$ modes of the GhMM with different transmission characteristics. In addition to being ultrabroadband and high-contrast, AT and AA features of the proposed GhMMs can be actively tuned by varying the chemical potential of graphene. Furthermore, it is shown that an onoff switching of AT factor at FIR and selective tunability at MIR frequencies can be obtained via varying $\mu$. Due to its subwavelength and planar configuration and active operation, these multilayer graphene-hBN metamaterials with AT and AA characteristics hold promise for integration with compact optical systems operating in the MIR and FIR ranges and are suitable for applications such as optical diodes, sensors, and thermal emitters.
\end{abstract}

Published under license by AIP Publishing. https://doi.org/10.1063/1.5118887

\section{INTRODUCTION}

Asymmetric light transmission $(\mathrm{AT})^{1-4}$ is known as the difference in transmission between the forward and backward direction of illuminations of a reciprocal electromagnetic device. AT has been an active research topic due to its potential applications in integrated photonic systems for communications and information processing, such as directional beam splitting, ${ }^{5}$ multiplexing, ${ }^{6}$ and optical interconnections. ${ }^{7}$ There are two approaches that can be employed to achieve a strong forward-to-backward transmission contrast in an optical system. The first approach is to break time-reversal symmetry, which can be realized in magneto-optical ${ }^{3}$ and nonlinear ${ }^{4}$ systems. The second approach requires breaking the spatial inversion symmetry. In this case, the transmission of nonpolarized and circularly/linearly polarized light through a device is Lorentz-reciprocal and can be realized in very diverse systems, e.g., chiral structures, ${ }^{1,8,9}$ metallic gratings, ${ }^{6,10}$ and dielectric-based meta-devices. ${ }^{11,12}$ Moreover, the combination of diffraction or subwavelength gratings with one- or two-dimensional photonic crystals, ${ }^{2,5,13}$ near-zero- index metastructures, ${ }^{14-17}$ and hyperbolic metamaterials (HMMs) ${ }^{18}$ can also lead to the observation of AT for linearly polarized light in the resulting hybrid structures.

By adding a properly designed diffraction grating on one side of a photonic crystal, ${ }^{2,13}$ it is possible to manipulate the wavevector 
of the incident light so that light couples to the supported modes by the system, which may result in waves propagating from one interface to the other due to the conservation of the tangential wavenumber. Therefore, the incident light that is reflected from the bare side of the photonic crystal can be injected inside, propagate through, and finally be extracted from the system once it is incident on the grating side. Later, it was suggested to use subwavelength gratings with evanescent diffraction orders. In this case, AT can be achieved owing to waves propagating parallel to the interfaces, which are associated with high- $\beta$ modes. ${ }^{18}$ High- $\beta$ modes are unique metamaterial states that can have wavevectors far exceeding the free-space wavevector $\left(\beta_{0}=\omega / c\right)$ and lie at the heart of many HMM-based device applications from imaging to quantum nanophotonics. ${ }^{19}$ AT for transverse magnetic (TM) waves at visible wavelengths has been demonstrated within this approach by using a passive HMM that consists of subwavelength metal/dielectric layers and is bounded by dissimilar gratings (labeled as " $A$ " and " $B$ " in Ref. 18). In that study, the bare HMM was designed to perform as a reflector within the wavelength range of interest in the visible range and to support high- $\beta$ modes. As explained in Ref. 18, the HMM and gratings in the above-mentioned structure are designed such that light is transmitted from the free space on side A into the free space on side B when the device is illuminated at normal incidence on side A. However, it is blocked from transmission into free space on side A when the device is illuminated at normal incidence on side B. For light incident on side A at a given frequency, the presence of grating A turns the incident light to a pair of high- $\beta$ propagating modes with tangential wavenumbers $\pm \beta$ larger in magnitude than $\beta_{0}$. Then, these propagating modes will be modified by grating $B$ such that they can be extracted from the structure, i.e., for the finally extracted light, we have $\left|\beta_{\text {out }}\right| \leq \beta_{0}$. Conversely, normally incident light on side B couples to a pair of nonpropagating, evanescent modes; thus, no light is extracted from the system.

Graphene $^{20}$ has found considerable scientific attention due to its incredible potential applications in optoelectronics ${ }^{21}$ and plasmonics. ${ }^{22}$ The surface conductivity of graphene $\left(\sigma_{g}\right)$ can be actively modulated via the tuning of its chemical potential $(\mu)$ through electrostatic/magnetostatic gating. ${ }^{20}$ For, $\operatorname{Im}\left(\sigma_{g}\right)>0$ graphene behaves like a very thin metal layer capable of supporting tunable TM surface plasmon polaritons. $^{23}$ Due to the tunable characteristics of this material, several studies have been recently conducted on AT in graphene-loaded gratings under a magnetic field, ${ }^{24}$ graphene metasurfaces, ${ }^{25-27}$ and metasurfaces integrated with graphene ${ }^{28,29}$ in the terahertz $(\mathrm{THz})$, far-infrared (FIR), and mid-infrared (MIR) ranges. However, for those studies, the operation frequency is limited to specific windows in the FIR and MIR ranges. An alternative way to achieve actively tunable AT, but in a wide range of operation, is to use multilayer graphenedielectric metamaterials. These metamaterials are planar structures comprising periodically arranged graphene sheets and subwavelengththickness dielectric layers that have been theoretically devised in the $\mathrm{THz}$ and FIR frequency regions, ${ }^{23,30-32}$ experimentally verified at the MIR frequencies, ${ }^{33}$ and can also be gated practically. ${ }^{34}$ Recently, considering effective parameters of a multilayer graphene-dielectric metamaterial in the calculations, dynamical tuning of AT was demonstrated at frequencies around $20 \mathrm{THz}{ }^{35}$ Another category of these artificial structures is multilayer graphene-hexagonal boron nitride (hBN) metamaterials (GhMMs) that consist of alternating layers of graphene and $\mathrm{hBN} .^{36,37} \mathrm{hBN}$ is a uniaxially anisotropic material that shows hyperbolic characteristics in the MIR range while acting as a dielectric medium at FIR frequencies. As a natural hyperbolic material, the dielectric constants of hBN are the same in the basal plane $\left(\varepsilon_{t} \equiv\right.$ $\left.\varepsilon_{x}=\varepsilon_{y}\right)$ but have opposite signs $\left(\varepsilon_{x} \varepsilon_{z}<0\right)$ in the normal direction $\left(\varepsilon_{z}\right)$ in the MIR region inside reststrahlen (RS) bands. ${ }^{38}$ The RS bands of hBN are categorized as type-I $\left(\varepsilon_{z}<0, \varepsilon_{t}>0\right)$ and type-II $\left(\varepsilon_{z}>\right.$ $\left.0, \varepsilon_{t}<0\right)$ according to their hyperbolic regime. Due to this property, finite-thickness slabs of hBN are capable of supporting subdiffractional volume-confined phonon polaritons for TM polarization. By combining graphene with $\mathrm{hBN}$ films in graphene-hBN heterostructures, one can also efficiently modify the hyperbolic dispersion of hBN phonons using graphene plasmons, i.e., hybrid plasmon-phonon polaritons (HPPs) are supported in this case. ${ }^{39,40}$ Due to the coupling between HPPs of each unit cell, high- $\beta$ HPP bands can be supported by a GhMM, ${ }^{37}$ which makes this structure very capable for AT purposes, as we demonstrate it here. Moreover, it has been recently shown that, due to the support of high- $\beta$ phonon polaritons, a bare film of hBN as a natural hyperbolic material can also be employed to achieve AT in a window in the MIR region. ${ }^{41}$

In the present paper, using a planar GhMM of subwavelength thickness that is bounded by two asymmetric gratings of Ge with an almost subwavelength period, we theoretically prove AT and asymmetric light absorption (AA) of TM-polarized light in the FIR and MIR ranges under normal illumination. Through analytical calculations based on the transfer matrix method (TMM), we first investigate the TM transmission characteristics of high- $\beta$ modes supported by the GhMM. Then, by appropriately designing the asymmetric gratings and thus exciting those high- $\beta$ propagating/evanescent modes in the GhMM, we obtain AT and AA for the TM polarization in an ultrabroadband range extending from 10 to $60 \mathrm{THz}$. Moreover, the tunable transmission and absorption characteristics of the suggested hybrid structures via changes in the chemical potential of the graphene layers are also investigated. It is shown that, by varying $\mu$, an on-off switching of asymmetric transmission factor (ATF)-i.e., from near-zero to nearly $40 \%$ at FIR-and a selective tunability at MIR frequencies can be obtained. To the best of our knowledge, this is the first study on hiring high- $\beta$ modes of graphene-hBN multilayer metamaterial to achieve actively tunable and broadband AT and AA in the FIR and MIR ranges.

\section{THEORETICAL BACKGROUND}

To investigate asymmetric transmission in the grating-bounded GhMM, we first need to gain an insight into the optical properties of a bare GhMM. The schematic of a bare GhMM is illustrated in the left panel of Fig. 1(a). As shown in this figure, the GhMM is composed of $N$ graphene multilayers that are separated by $N-1 \mathrm{hBN}$ films of thickness $d$. Each graphene multilayer consists of $N_{g}$ graphene sheets. Considering hBN as a uniaxially anisotropic medium with permittivity tensor $\varepsilon_{h B N}=\operatorname{diag}\left(\varepsilon_{t}, \varepsilon_{t}, \varepsilon_{z}\right),{ }^{38}$ where

$$
\varepsilon_{m}=\varepsilon_{\infty, m} \times\left[1+\frac{\omega_{L O, m}^{2}-\omega_{T O, m}^{2}}{\omega_{T O, m}^{2}-\omega^{2}-i \omega \Gamma_{m}}\right], \quad m=t, z,
$$

and applying boundary conditions appropriately, we arrive at the 

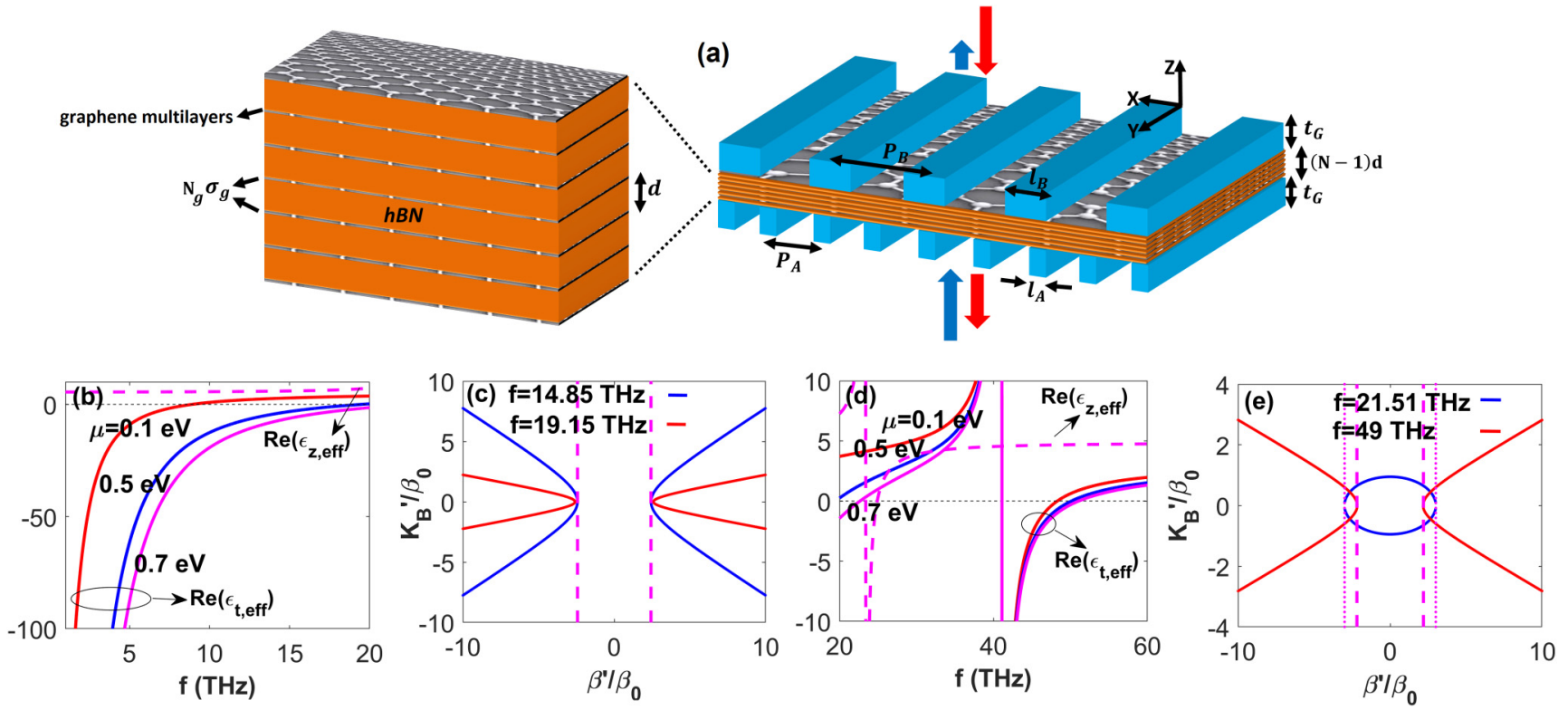

FIG. 1. (a) Schematics of the graphene-hBN metamaterial (left) and the grating-bounded GhMM (right). As seen from the left schematic, in the GhMM, the hBN layers of thickness $d$ are separated by graphene multilayers each composed of $N_{g}$ graphene sheets. The total number of hBN layers (graphene multilayers) is $N-1(N)$, as depicted in the left diagram of panel (a). Panels (b) and (d), respectively, represent the FIR and MIR EMT-derived real part of the " $z$ " and tangential components of the effective permittivity of the GhMM, obtained from Eq. (3), for $\mu=0.1 \mathrm{eV}$ (red curves), $\mu=0.5 \mathrm{eV}$ (blue curves), and $\mu=0.7 \mathrm{eV}$ (pink curves). Panels (c) and (e) illustrate the EMT-derived dispersion curves of the GhMM at four different frequencies in the FIR and MIR ranges, respectively.

following dispersion relation of the GhMM for the TM polarization: ${ }^{37}$

$$
\cos \left(K_{B} d\right)=\cos \left(k_{h} d\right)+\alpha k_{h} / 2 \varepsilon_{t} \sin \left(k_{h} d\right),
$$

where $K_{B}$ is the complex Bloch wavenumber, $k_{h}=$ $\sqrt{\varepsilon_{t}\left(\varepsilon_{z} \beta_{0}^{2}-\beta^{2}\right) / \varepsilon_{z}}, \quad \beta=k_{x} \quad$ (i.e., in general, $\left.\beta^{\prime}+i \beta^{\prime \prime}\right)$, and $\alpha=N_{g} \sigma_{g} / i \omega \epsilon_{0} . \omega_{T O, m}$ and $\omega_{L O, m}$ denote transverse and longitudinal optical phonon frequencies, respectively, and $\Gamma_{m}$ is the loss factor. The optical conductivity of the graphene $\left(\sigma_{g}=\sigma_{g}^{\text {intra }}+\right.$ $\sigma_{g}^{\text {inter }}$ ) can also be formulated as ${ }^{23}$

$$
\begin{gathered}
\sigma_{g}^{\text {intra }}(\omega)=\frac{e^{2}}{4 \hbar} \frac{i}{2 \pi}\left\{\frac{16 k_{B} T}{\hbar \Omega} \ln \left(2 \cosh \left(\frac{\mu}{2 k_{B} T}\right)\right)\right\}, \\
\sigma_{g}^{\text {inter }}(\omega)=\frac{e^{2}}{4 \hbar}\left\{\frac{1}{2}+\frac{1}{\pi} \arctan \left(\frac{\hbar \Omega-2 \mu}{2 k_{B} T}\right)-\frac{i}{2 \pi} \ln \frac{(\hbar \Omega+2 \mu)^{2}}{(\hbar \Omega-2 \mu)^{2}+\left(2 k_{B} T\right)^{2}}\right\},
\end{gathered}
$$

and $\Omega=\omega+i \tau^{-1}$ where $\tau$ is the relaxation time of electrons, $k_{B}$ is the Boltzmann constant, and $T$ is the temperature.

As mentioned earlier, the hBN layers in the graphene-hBN multilayer metamaterial have subwavelength thicknesses. Therefore, by applying the effective medium theory (EMT) on Eq. (2), it is also possible to evaluate the optical response of the GhMM by considering the metamaterial as a uniaxially anisotropic medium with permittivity $\varepsilon_{G h M M}=\operatorname{diag}\left(\varepsilon_{t, \text { eff }}, \varepsilon_{t, \text { eff }}, \varepsilon_{z, \text { eff }}\right)$ with the following dispersion relation:

$$
\frac{K_{B}^{2}}{\varepsilon_{t, e f f}}+\frac{\beta^{2}}{\varepsilon_{z, e f f}}=\beta_{0}^{2},
$$

where $\varepsilon_{t, e f f}=\varepsilon_{t}-\frac{\alpha}{d}$ and $\varepsilon_{z, \text { eff }}=\varepsilon_{z}$. This EMT description can give us a fair, but not exact, insight into the optical response of the GhMM for small values of $\beta .^{36}$ For large values of the 
wavenumber, exact descriptions-using Eq. (2) or the transfer matrix method (TMM) ${ }^{37}$-should be considered. As mentioned, employing TMM calculations can be an alternative approach for the exact investigation of the optical response of the graphene-hBN metamaterial. In our calculations, as mentioned above, we suppose that the GhMM is bounded by a nonmagnetic medium with permittivity $\varepsilon_{A}$ and consists of $N$ graphene multilayers, i.e., $(N-1) \mathrm{hBN}$ layers, and the first layer of the metamaterial is placed at $z=0$. By applying the TM boundary conditions on

$$
H_{y}(z)=\left\{\begin{array}{ll}
H_{i} e^{i k_{A} z}+H_{r} e^{-i k_{A} z}, & z<0 \\
H_{n 1} e^{i k_{h}[z-(n-1) d]}+H_{n 2} e^{-i k_{h}[z-(n-1) d]}, & (n-1) d<z<n d \\
H_{r} e^{i k_{A}(z-n d)}, & z>n d
\end{array}\right\}
$$

where $n=1,2, \ldots, N$, we arrive at $\Im=|t|^{2}$ as an analytical relation for transmission of the metamaterial with $t=1 / M_{11}$ and $M=\left[\begin{array}{l}M_{11} \\ M_{12}\end{array}\right]=m_{A 1}^{-1}\left(m_{1} m_{2}^{-1}\right)^{N} m_{A 2}$. Here,

$$
\begin{gathered}
m_{A 1}=\left[\begin{array}{cc}
i k_{A} / \varepsilon_{A} & -i k_{A} / \varepsilon_{A} \\
1-i \alpha k_{A} / \varepsilon_{A} & 1+i \alpha k_{A} / \varepsilon_{A}
\end{array}\right], \\
m_{A 2}=\left[\begin{array}{c}
i k_{A} / \varepsilon_{A} \\
1
\end{array}\right], \quad k_{A}=\sqrt{\varepsilon_{A} \beta_{0}^{2}-\beta^{2}}, \\
m_{1}=\left[\begin{array}{cc}
i k_{h} / \varepsilon_{t} & -i k_{h} / \varepsilon_{t} \\
1 & 1
\end{array}\right]
\end{gathered}
$$

and

$$
m_{2}=\left[\begin{array}{cc}
i k_{h} e^{i k_{h} d} / \varepsilon_{t} & -i k_{h} e^{-i k_{h} d} / \varepsilon_{t} \\
\left(1-i \alpha k_{h} / \varepsilon_{t}\right) e^{i k_{h} d} & \left(1+i \alpha k_{h} / \varepsilon_{t}\right) e^{-i k_{h} d}
\end{array}\right]
$$

\section{RESULTS AND DISCUSSION}

In this section, we represent our findings by categorizing the results in a window of the far-IR region $(10-20 \mathrm{THz})$ and in the mid-IR $(20-60 \mathrm{THz})$ range. In each subsection, first, using the TMM, the resonant transmission bands of a bare metamaterialbounded with air without a grating-are investigated. Then, the grating-bounded metamaterial is designed based on the information extracted from the transmission bands. Schematic of the gratingbounded GhMM is depicted in the right panel of Fig. 1(a). It is noteworthy that the investigation of grating-bounded structures can be achieved experimentally owing to the current fabrication techniques. ${ }^{18}$ Notice that the GhMMs used in our designs are the same for operation in the far-IR and mid-IR ranges (i.e., $d=100 \mathrm{~nm}, N_{g}=10$, and $N=11$ ), while the dimensions of the gratings " $\mathrm{A}$ " and " $\mathrm{B}$ " differ for operation in different frequency regions. Moreover, here we refer to $T_{A \rightarrow B}$ and $T_{B \rightarrow A}$ as forward $\left(T_{F}\right)$ and backward $\left(T_{B}\right)$ transmissions, respectively. The parameters in the calculations are taken as $T=300 \mathrm{~K}, \tau=0.2 \mathrm{ps}, \mu=0.5 \mathrm{eV}$ (otherwise stated), $\varepsilon_{\infty, x}=2.95$, $\varepsilon_{\infty, z}=4.87, \quad \omega_{L O, x} / 2 \pi=48.266 \mathrm{THz}, \quad \omega_{T O, x} / 2 \pi=41.071 \mathrm{THz}$, $\omega_{L O, z} / 2 \pi=24.882 \mathrm{THz}, \omega_{T O, z} / 2 \pi=23.383 \mathrm{THz}, \Gamma_{x} / 2 \pi=0.119 \mathrm{THz}$, $\Gamma_{z} / 2 \pi=0.149 \mathrm{THz}$, and $t_{G}=1 \mu \mathrm{m}$. Therefore, considering a separation distance of the graphene layers in each graphene multilayer of $3.3 \AA{ }^{23}$ the total subwavelength thickness of the grating-bounded GhMMs in this paper is almost $3 \mu \mathrm{m}$ (the thickness of the bare GhMM is almost $1 \mu \mathrm{m}$ ). The grating material is chosen to be Ge, which shows extremely low losses in the far- and mid-IR ranges and can increase the efficiency of the AT device compared with the use of lossy materials.

To gain an understanding of the EMT-derived optical response of the bare GhMM, $\varepsilon_{t, \text { eff }}$ (solid lines) and $\varepsilon_{z, \text { eff }}$ (dashed line) of the metamaterial as a function of frequency for three different values of the chemical potential of the graphene layers are represented in the far- and mid-IR ranges in panels (b) and (d) of Fig. 1, respectively. Moreover, the dispersion curves of the proposed metamaterial at two typical frequencies are illustrated in panels (c) and (e) of Fig. 1. As shown in Fig. 1(b), $\boldsymbol{\varepsilon}_{z, \text { eff }}>0$ in the FIR range, while $\boldsymbol{\varepsilon}_{t, \text { eff }}$ (solid lines) can have tunable negative values depending on different values of the chemical potential of the graphene layers. Therefore, the structure acts as a type-II hyperbolic metamaterial, depending on the frequency and chemical potential. As an example, Fig. 1(c) illustrates type-II hyperbolic equifrequency dispersion contours of the metamaterial at $f=14.85 \mathrm{THz}$ and $19.15 \mathrm{THz}$, indicating propagation only for the modes with wavevectors having $\left|\beta^{\prime}\right| \geq \beta_{c}$. Here, $\beta_{c}= \pm \operatorname{Re}\left(\sqrt{\varepsilon_{z, e f f}}\right) \beta_{0}$ represents the cutoff wavevectors, which are illustrated by the vertical dashed lines at $\pm 2.43 \beta_{0}$. In the MIR range, due to the intrinsic hyperbolic response of $\mathrm{hBN},{ }^{39} \mathrm{GhMM}$ shows a more complex response than the one observed at FIR frequencies. It is seen from panel (d) of Fig. 1 that, first, $\varepsilon_{t, e f f}$ can be tuned by modifying the chemical potential of the graphene layers, while $\varepsilon_{z, \text { eff }}$ is unaffected [the same feature is observed in the FIR range, Fig. 1(b) dashed line]. Second, as a typical example, for the case of $\mu=0.5 \mathrm{eV}$, GhMM acts as a type-II (type-I) hyperbolic metamaterial within the $40.1<f(\mathrm{THz})<50.1[23.4<f(\mathrm{THz})<24.9]$ range and effectively behaves as a uniaxially anisotropic medium with elliptical dispersion at the other MIR frequencies. EMT-derived dispersion curves of the metamaterial at $f=21.51 \mathrm{THz}$ and $49 \mathrm{THz}$ are visualized in Fig. 1(e). The metamaterial exhibits an elliptical (type-II hyperbolic) response at the former (latter) frequency with a cutoff wavenumber of $\pm 3 \beta_{0}\left( \pm 2.18 \beta_{0}\right)$.

\section{A. Far-infrared tunable asymmetric light transmission and absorption}

To investigate the AT response of the grating-bounded GhMM that is depicted in the right panel of Fig. 1(a), we first need 

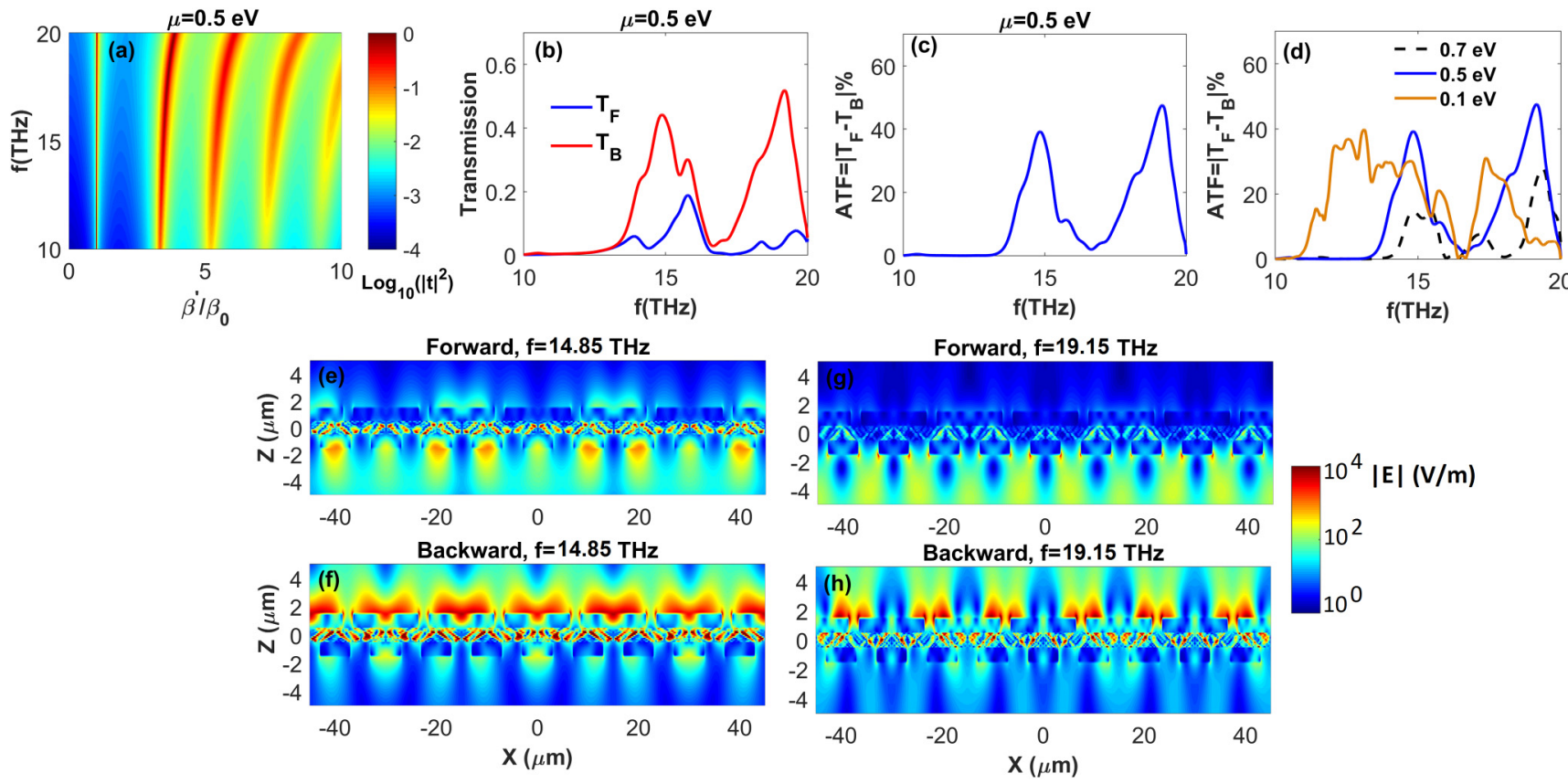

FIG. 2. FIR transmission characteristics of the bare and grating-bounded GhMM for $P_{B}=15 \mu \mathrm{m}, I_{B}=13 \mu \mathrm{m}, P_{A}=10 \mu \mathrm{m}$, and $I_{A}=6 \mu \mathrm{m}$. (a) TMM-calculated TM-polarized light transmission, on a logarithmic scale, as a function of frequency and normalized wavenumber for a free-standing GhMM. (b) TM-polarized light transmission through the grating-bounded metamaterial for normally incident forward (blue curve) and backward (red line) directions of illumination at $\mu=0.5 \mathrm{eV}$. Panel (c) represents the asymmetric transmission factor, ATF, of the device for the case of panel (b). The ATFs of the grating-bounded GhMM at $\mu=0.1 \mathrm{eV}$ (solid brown line), $\mu=0.5 \mathrm{eV}$ (solid blue line), and $\mu=0.7 \mathrm{eV}$ (dashed black line) are shown in panel (d). Panels (e)-(h) show the electric field mode profiles of normally incident TM-polarized light for forward and backward directions of illumination at the peaks of the ATF observed in panel (c).

to gain insight into the transmission characteristics of a bare GhMM. Using the TMM, Fig. 2(a) represents the TM transmission of the electromagnetic field, $|t|^{2}$, through a bare GhMM at FIR frequencies as a function of $\beta^{\prime} / \beta_{0}$ in the positive-half of the $\beta$ space.

In agreement with the transmission bandgap predicted by the EMT, small values of $|t|^{2}$ are observed at $\beta^{\prime} / \beta_{0}<2.5$, while by increasing $\beta^{\prime}$, the transmission rises and falls due to the support of high- $\beta$ modes by the metamaterial. As similarly observed in layered metal-dielectric metamaterials, ${ }^{18}$ these TMM-calculated fluctuations [also verified by the finite difference time domain method $(\text { FDTD })^{42}$ at normal incidence] that are not predicted by EMT are a sign of the breakdown of the homogenization approximation and the consideration of finite periods in the TMM calculations. The appearance of high- $\beta$ multiple transmission bands observed in Fig. 2(a) leads to a multifunctional capability of the graphene-hBN metamaterial for AT devices compared to the metallic metamaterials. Taking $P_{B}=15 \mu \mathrm{m}, l_{B}=13 \mu \mathrm{m}, P_{A}=10 \mu \mathrm{m}$, and $l_{A}=6 \mu \mathrm{m}$ (leading to fundamental reciprocal lattice wavevectors of magnitude $G_{A}=2 \pi / P_{A}$ and $\left.G_{B}=2 \pi / P_{B}\right)$ as the grating parameters, we investigate AT of the grating-bounded GhMM in the $10-20 \mathrm{THz}$ window in the FIR region. By these choices and employing the grating law $\left(2 \pi / \lambda \sin (\theta)=\beta+n G_{A, B}\right), G_{A}$ and $G_{B}$ can couple normally incident light $(\theta=0)$ into a high- $\beta$ propagating/evanescent wave in the GhMM having a transverse wavevector located inside the $\beta$ space with high/low transmission in Fig. 2(a). Here, $\theta$ is the angle of incident light with respect to the $+Z /-Z$ direction for forward/backward illumination, and $n= \pm 1, \pm 2, \ldots$. Similar to the mechanisms leading to AT for the metallic metamaterials, ${ }^{18}$ there are two conditions responsible for achieving AT. First, to enable the coupling of the wave incident on the metamaterial from side A (B) to an outgoing wave on side B (A) (having tangential wavevector $\beta_{\text {out }}$ ), $G_{A}$ and $G_{B}$ must satisfy the condition $\left|G_{A}-G_{B} / n\right|=\beta_{\text {out }}<\beta_{0}$. Second, to reach a lower transmission in the forward direction than in the backward one at each frequency, $G_{A}$ and $2 G_{A}$ must be placed inside the low-transmission regions $\left(|t|^{2}<0.01\right)$ in Fig. 2(a). The resulting forward and backward transmissions through the grating-bounded metamaterial for normal incidence are shown in Fig. 2(b) by solid blue and red lines, respectively. It is observed from this figure that there is a distinguishable difference between $T_{F}$ and $T_{B}$, i.e., higher values of transmission are obtained for forward illumination compared with the backward one. Moreover, the ATF of the device, which can be defined as $\left|T_{F}-T_{B}\right| \times 100 \%$, is illustrated in Fig. 2(c). Notice that the two broad resonances obtained in $T_{B}$ at 14.85 and $19.15 \mathrm{THz}$ [Fig. 2(b)] lead to strong peaks in the ATF, with values of almost $40 \%$ and $50 \%$, respectively. It is noteworthy that at $f=14.85 \mathrm{THz}(19.15 \mathrm{THz})$, the corresponding obtained values of $G_{A}$ and $G_{B}$ are $2.02 \beta_{0}\left(1.57 \beta_{0}\right)$ and $\sim 2 \times 1.34 \beta_{0}\left(\sim 4 \times 1.04 \beta_{0}\right)$, respectively, and fully follow the above-mentioned conditions for achieving AT with $T_{B}>T_{F}$. In addition to having a broadband 
response in the FIR region, having tunable characteristics through modifying the chemical potential of the graphene layers is another distinguishable feature of the structure under our consideration in this paper. As observed from Fig. 2(d), by changing $\mu$ from 0.1 to $0.7 \mathrm{eV}$, it is possible to obtain AT with high ATF values at frequencies of interest within $10-20 \mathrm{THz}$. In other words, by varying the chemical potential of the graphene layers, we can obtain on-off switching of AT, i.e., from near-zero ATF to nearly $40 \%$ efficiency. As a proof of concept, the electric field mode profiles of TM-polarized plane waves passing through the grating-bounded GhMM at the above-mentioned frequencies for forward and backward illuminations are shown in Figs. 2(e)-2(h). As clearly observed, in support of Fig. 2(b), the plane wave passes more strongly through the metamaterial with different patterns for backward illumination compared with the forward one. It is noteworthy that for the FDTD simulations, the material data are used for $\mathrm{Ge}$ and $\mathrm{Au}$, and graphene is defined as a $2 \mathrm{D}$ sheet with the optical conductivity provided by Eq. (3). Moreover, hBN permittivity is introduced to the used software as data files. Furthermore, a unit cell with periodic boundary condition in the $\mathrm{X}$ direction and a perfectly matched layer along the $Z$ axis are employed, and the structure is excited by a broadband plane wave.

Another characteristic of the grating-bounded GhMM is the support of nearly perfect absorption resonances with asymmetric features, i.e., absorption $A=1-(T+R)$, where $T$ and $R$ mean, respectively, transmission and reflection, may differ for the forward $\left(A=A_{F}\right)$ and the backward $\left(A=A_{B}\right)$ illuminations. AT and AA coexist in the studied structure, so it is also worth considering the main features of AA. Moreover, investigating the absorption feature of the structure is also an attempt to more uncover the mechanism behind the AT response. Figure 3 shows FDTD-calculated absorption characteristics of the metamaterial, $A_{F}$ and $A_{B}$, for normally incident light and three different values of $\mu$.

It is seen from panels (a) to (c) of Fig. 3 that the metamaterial is capable of possessing the AA of light depending on the directions of illumination. Based on the broken spatial inversion symmetry, a similar AA behavior has also been observed in the metallic ${ }^{43}$ and polar-dielectric based photonic crystals ${ }^{44}$ and few-layer structures. ${ }^{45}$ The absorption spectrum is considerably tunable through changes in $\mu$. This feature allows us to switch between the case when asymmetry in absorption is very weak $(\mu=0.1 \mathrm{eV})$ to the case when the threefold difference between $A_{F}$ and $A_{B}$ can be achieved $(\mu=0.7 \mathrm{eV})$, in the vicinity of $14 \mathrm{THz}$. Moreover, it is observed that for larger values of $\mu$, absorption shows a sharper resonant behavior with the near-unity magnitude feature. This perceptible aspect makes the proposed metamaterial very capable for FIR sensing applications. Moreover, by comparing Figs. 3(b) and 2(b), it is understood that the structure is mostly reflective at $f<13.5 \mathrm{THz}$, while at larger frequencies, both transmittive and absorptive features of the structure take the lead for the observation of AT and AA responses.

\section{B. Mid-infrared tunable asymmetric light transmission and absorption}

In this subsection, we focus on the investigation of tunable AT at MIR frequencies. In Fig. 4(a), we first analyze the TMMcalculated transmission characteristic of the bare graphene-hBN metamaterial. As mentioned earlier, it is well known that a film of
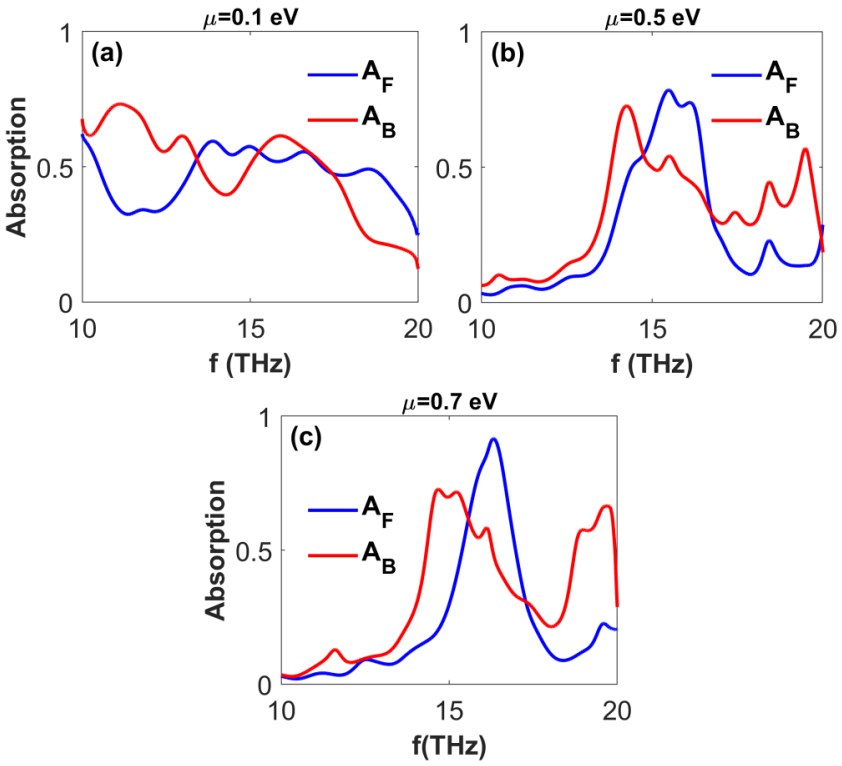

FIG. 3. FIR absorption curves of the grating-bounded GhMM for $P_{B}=15 \mu \mathrm{m}$, $I_{B}=13 \mu \mathrm{m}, P_{A}=10 \mu \mathrm{m}$, and $I_{A}=6 \mu \mathrm{m}$ at $\mu=0.1 \mathrm{eV}$ [panel (a)], $\mu=$ $0.5 \mathrm{eV}$ [panel (b)], and $\mu=0.7 \mathrm{eV}$ [panel (c)] for forward (blue lines) and backward (red lines) illuminations of normally incident TM-polarized light.

hBN has a hyperbolic phononic response in the MIR region, and its combination with graphene in graphene-hBN heterostructures leads to the support of some new modes created inside and outside of the RS bands of hBN, named as HPPs. ${ }^{39}$ As it was shown recently, ${ }^{37}$ due to the hybridization of plasmon-phonon modes of each unit cell in the GhMM, the resulting metamaterial supports HPP bands with high- $\beta$ propagation characteristics. Here, we employ the high- $\beta$ modes to achieve asymmetric transmission and absorption with tunable features.

Due to the support of several hybrid propagating/evanescent bands at different high values of wavenumber in the MIR region [see Fig. 4(a)], the bare GhMM is a versatile candidate for obtaining AT. Note that the dashed lines in this figure (and also in the other panels of Fig. 4) represent the edges of the RS bands of the GhMM. It is noteworthy that more investigations on the bare metamaterial using TMM reveal the point that, as expected, by considering more periods of the metamaterial in the calculations, the results will be in better agreement with the ones predicted by EMT in panels (d) and (e) of Fig. 1. However, in practice, a limited number of periods can be utilized. Thus, as mentioned earlier, we have considered $N=11$ in our calculations. The required conditions to obtain AT using the GhMM at MIR frequencies are similar to those in the FIR range, i.e., first $\left|G_{A} / n-G_{B}\right|<\beta_{0}$ and second $G_{A}$ $\left(G_{B}\right)$ and $2 G_{A}\left(2 G_{B}\right)$ must be placed inside the low-transmission regions $\left(|t|^{2}<0.03\right)$ in Fig. 4(a) to achieve a lower transmission in the forward (backward) direction than in the backward (forward) one at each frequency. Note that, in this frequency range, different from the results shown in Sec. III A, both $T_{F}>T_{B}$ and $T_{B}>T_{F}$ results can be obtained. Taking $P_{B}=5 \mu \mathrm{m}, l_{B}=3 \mu \mathrm{m}, P_{A}=3 \mu \mathrm{m}$, 

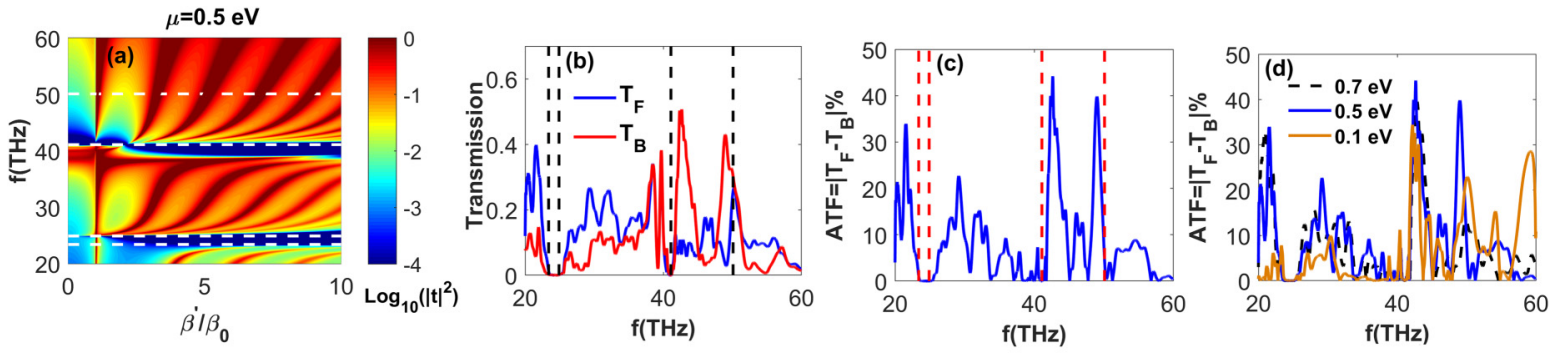

Forward, $\mathrm{f}=\mathbf{2 1 . 5 1} \mathrm{THz}$
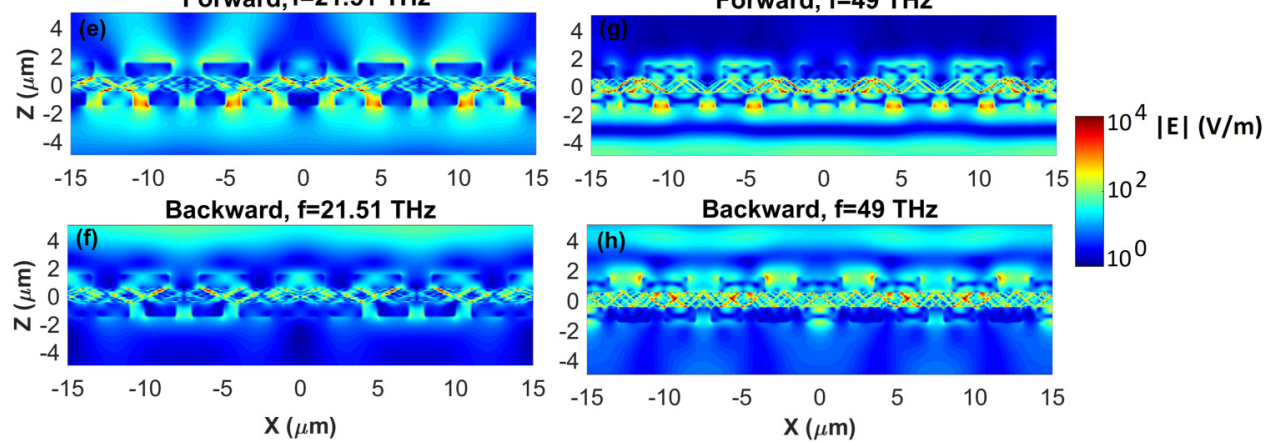

FIG. 4. MIR transmission characteristics of the bare and the grating-bounded GhMM for $P_{B}=5 \mu \mathrm{m}, I_{B}=3 \mu \mathrm{m}, P_{A}=3 \mu \mathrm{m}$, and $I_{A}=2 \mu \mathrm{m}$. (a) Logarithmic scale transmission of TM-polarized light through a free-standing bare GhMM calculated by TMM. (b) The transmission of forwardly illuminated (blue curve) and backwardly illuminated (red curve) TM-polarized light through the grating-bounded GhMM for normal incidence at $\mu=0.5 \mathrm{eV}$. The ATF of the device for the case of panel (b) is illustrated in panel (c). In panel (d), solid brown $(\mu=0.1 \mathrm{eV})$, solid blue $(\mu=0.5 \mathrm{eV})$, and dashed black lines $(\mu=0.7 \mathrm{eV})$ represent the ATF of the device for three different values of the chemical potential of the graphene layers. The electric field profiles of normally incident TM-polarized light for forward and backward illuminations at two typical resonant peaks of panel (c) are shown in panels (e)-(h). Note that the dashed lines in panels (a)-(c) highlight the edges of the RS bands of GhMM.

and $l_{A}=2 \mu \mathrm{m}$, Fig. 4 (b) numerically shows the AT of the gratingbounded GhMM within the MIR range. It is observed from this figure that for $37.15<f(\mathrm{THz})<45.08$ and $47.75<f(\mathrm{THz})<$ $51.29, T_{B}>T_{F}$, while for the other frequencies, the $T_{F}>T_{B}$ condition is achieved. It should be highlighted that AT is observed inside and outside of the type-II band of the metamaterial, excluding the type-I band. This point indicates that both the phononic and plasmonic characteristics of the high $\beta$ modes supported by the bare GhMM contribute to the mechanism behind the AT phenomena. The multiresonant characteristic observed in the AT, which is a consequence of the highly dispersive transmission feature of the bare GhMM, leads to the observation of large ATF values in the system, as depicted in Fig. 4(c). As seen in this figure, the modes supported inside and outside of the type-II band show a noticeable increase in the ATF values of up to almost $45 \%$. As two typical examples, notice the resonances at $f=21.51$ and $49 \mathrm{THz}$. At the former (latter) frequency, the obtained values of $G_{A}$ and $G_{B}$ are $2 \times 2.3 \beta_{0}\left(2.04 \beta_{0}\right)$ and $2.8 \beta_{0}\left(1.22 \beta_{0}\right)$, respectively, and are in support of the $T_{F}>T_{B}\left(T_{B}>T_{F}\right)$ results shown in Fig. $4(\mathrm{~b})$. The tunable characteristic of the ATF of the grating-bounded GhMM in the MIR region by modifying the chemical potential of the graphene layers is also shown in Fig. 4(d) for three different values of $\mu$. As observed, both the strength and spectral location of the plasmonic and phononic resonances outside and inside the type-II band can
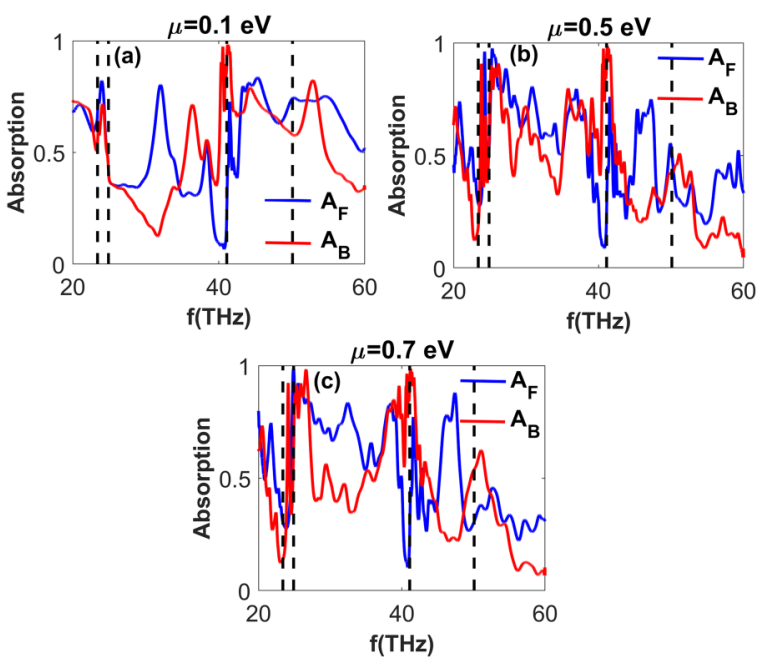

FIG. 5. MIR absorption plots of the grating-bounded GhMM for $P_{B}=5 \mu \mathrm{m}$, $I_{B}=3 \mu \mathrm{m}, P_{A}=3 \mu \mathrm{m}$, and $I_{A}=2 \mu \mathrm{m}$ at three different values of the chemical potential of the graphene layers for normally incident light for forward and backward directions of illumination. Similar to Fig. 4, the vertical dashed lines represent the edges of the RS bands of GhMM. 

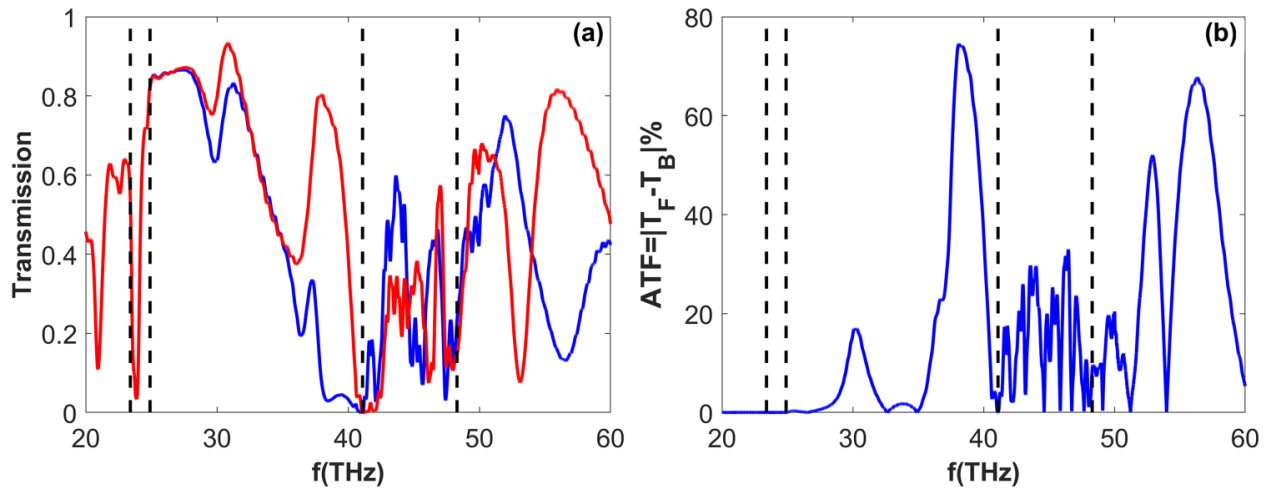

FIG. 6. Similar to panels (b) and (c) of Fig. 4 while here MIR transmission [panel (a)] and ATF [panel (b)] of the grating-bounded GhMM with $d=50 \mathrm{~nm}$, $P_{B}=4 \mu \mathrm{m}, I_{B}=2 \mu \mathrm{m}, P_{A}=3 \mu \mathrm{m}$, and $I_{A}=2 \mu \mathrm{m}$ are illustrated. be considerably controlled, excluding the type-I band in which AT is not observed. Moreover, there are frequency ranges of weak and strong sensitivities to the variations of $\mu$, so selective tuning of the asymmetric transmission factor can be achieved, i.e., the different regions with high ATF can be controlled separately. The spatial distributions of the electric field at the two above-mentioned frequencies for forward and backward illuminations are illustrated in Figs. 4(e)-4(h). As observed, light transmission for forward illumination is stronger than that of the backward case at $f=21.51 \mathrm{THz}$, while the opposite behavior is observed for illumination at $f=49 \mathrm{THz}$. In order to obtain more insights into the MIR AT behavior of the grating-bounded GhMM, FDTD-calculated absorption plots for three different values of the chemical potential of graphene layers and two directions of illumination are shown in Fig. 5.

Some noticeable points regarding the absorption plots, shown in Fig. 5, can be highlighted here: (i) the metamaterial shows broad band AA with tunable characteristics; thus, the suggested designs here can find potential application as active metamaterial light absorbers; ${ }^{46}$ (ii) the support of multiresonant absorption peaks make the structure beneficial for sensing applications; (iii) the appearance of the resonant absorption peaks inside type-II $(40.1<f(\mathrm{THz})<50.1)$ and type-I $(23.4<$ $f(\mathrm{THz})<24.9)$ bands is an indication of the support of hybrid plasmonic-phononic modes of both hyperbolicity by the metamaterial. Note that the appearance of the nearly perfect absorption resonances observed in Fig. 5(b) are in support of almost zero transmission values obtained in the $23.4<f(\mathrm{THz})<25.6$ frequency range for forward (backward) illumination in Fig. 4(b). More investigations reveal that, for the case of $\mu=0.5 \mathrm{eV}$, light is trapped and absorbed inside the metamaterial for forward illumination due to the excitation of the above-mentioned hybrid resonant modes around $f=24.28$ and $25.33 \mathrm{THz}(f=23.84,24.38$, and $25.21 \mathrm{THz}$ for backward illumination), and it is mostly reflected at the other frequencies in the above-mentioned frequency range. Similarly, the same mechanism is responsible for almost zero $T_{F}$ and $T_{B}$ within $40.69<f(\mathrm{THz})<41.38$, which is observed in Fig. 4(b).

At the end, some important points should be highlighted: (i) We have also examined the results for different values of the relaxation time of graphene (i.e., $\tau=0.1 \mathrm{ps}, 0.4 \mathrm{ps}$, and $0.8 \mathrm{ps}$ ) and the case for which single layers of graphene $\left(N_{g}=1\right)$ is replaced with the graphene multilayers $\left(N_{g}=10\right)$ in the calculations. It has been found out that the results are valid for different values of $\tau$ (even
ATF can be increased for $\tau=0.4 \mathrm{ps}$ and $0.8 \mathrm{ps}$ ), and for the case of $N_{g}=1$, ATF values are decreased to $20 \%(40 \%)$ in the FIR (MIR) region. (ii) Because of the symmetry of the presented designs, i.e., employing $1 \mathrm{D}$ gratings in the calculations, they effectively function at TM polarization. However, by replacing the 1D gratings with appropriately designed 2D gratings (e.g., the ones based on circular disks), it is possible to achieve the asymmetric optical responses for both TE and TM polarization. (iii) The above-taken geometrical parameters were optimally chosen to achieve broadband AT with maximum contrast difference for the case of $d=100 \mathrm{~nm}$. As we have investigated recently, ${ }^{37}$ considering different values of the hBN thickness in the calculations, high $\beta$ propagating/evanescent HPP modes can be supported at different frequencies and values of the wavenumber, compared with the case of $d=100 \mathrm{~nm}$. Thus, we have also analyzed the results for two additional values of the hBN thickness, i.e., $d=50 \mathrm{~nm}$ and $d=150 \mathrm{~nm}$. As illustrated in Fig. 6, taking $d=50 \mathrm{~nm}$ and optimizing the geometrical parameters as $P_{B}=4 \mu \mathrm{m}, l_{B}=2 \mu \mathrm{m}$, $P_{A}=3 \mu \mathrm{m}$, and $l_{A}=2 \mu \mathrm{m}$, it is possible to increase ATF of the grating-bounded GhMM up to $75 \%$ in the MIR region.

\section{CONCLUSIONS}

In conclusion, we have analytically studied the transmission characteristic of a bare GhMM and numerically designed quasiplanar devices based on this metamaterial, which show asymmetric absorption and high-contrast asymmetric transmission characteristics in the MIR and FIR frequency ranges. The AT factor of the suggested devices reaches $50 \%$ once the thickness of the hBN layers in the metamaterial is taken to be as $100 \mathrm{~nm}$, and it can reach up to $75 \%$ in the MIR region for $d=50 \mathrm{~nm}$. The GhMMs consist of alternating layers of graphene multilayers (11 layers) and hBN films (10 layers) and are bounded with two asymmetric Ge gratings with subwavelength periodicities. The total subwavelength thickness of the planar grating-bounded GhMM devices makes the designs promising for integration with compact optical systems. The mechanism behind AT here is similar to that of AT studied earlier in the visible region using a passive device, which is based on the excitation of different propagating or evanescent high- $\beta$ modes supported by an HMM. ${ }^{18}$ However, in the present study, the AT and AA characteristics are actively tunable via modifying $\mu$ of the graphene layers, and they are observed in a wide range of frequencies extending from FIR to MIR region. More specifically, it is possible to achieve on-off switching of 
ATF at FIR and selective tunability at MIR frequencies. Having higher contrast and considerably broader operation range are the most advantageous characteristics of these devices compared to recently investigated ones. ${ }^{41}$ The proposed designs in this paper can find potential applications such as actively tunable optical diodes, sensors, light absorbers, ${ }^{46}$ and thermal emitters for the operation at MIR and FIR frequencies.

\section{ACKNOWLEDGMENTS}

The authors acknowledge support from TUBITAK (Nos. 113E331, 114E374, and 115F560) and Narodowe Centrum Nauki (NCN), Poland (DEC-2015/17/B/ST3/00118-Metasel). E.O. also acknowledges partial support from the Turkish Academy of Sciences.

\section{REFERENCES}

${ }^{1}$ V. A. Fedotov, P. L. Mladyonov, S. L. Prosvirnin, A. Rogacheva, Y. Chen, and N. I. Zheludev, Phys. Rev. Lett. 97, 167401 (2006).

${ }^{2}$ A. E. Serebryannikov, T. Magath, and K. Schuenemann, Phys. Rev. E 74, 066607 (2006).

${ }^{3}$ L. Bi, J. Hu, P. Jiang, D. H. Kim, G. F. Dionne, L. C. Kimerling, and C. A. Ross, Nat. Photonics 5, 758-762 (2011).

${ }^{4}$ M. Scalora, J. P. Dowling, C. M. Bowden, and M. J. Bloemer, J. Appl. Phys. 76, 2023-2026 (1994).

${ }^{5}$ E. Colak, A. E. Serebryannikov, P. V. Usik, and E. Ozbay, J. Appl. Phys. 119, 193108 (2016).

${ }^{6}$ S. Cakmakyapan, A. E. Serebryannikov, H. Caglayan, and E. Ozbay, Opt. Express 20, 26636-26648 (2012).

${ }^{7}$ L. Fan, J. Wang, L. T. Varghese, H. Shen, B. Niu, Y. Xuan, A. M. Weiner, and M. Qi, Science 335, 447-450 (2012).

${ }^{8}$ S. Fang, K. Luan, H. F. Ma, W. Lv, Y. Li, Z. Zhu, C. Guan, J. Shi, and T. J. Cui, J. Appl. Phys. 121, 033103 (2017).

${ }^{9}$ L. Stephen, N. Yogesh, and V. Subramanian, J. Appl. Phys. 123, 033103 (2018).

${ }^{10}$ B. Tang, Z. Li, Z. Liu, F. Callewaert, and K. Aydin, Sci. Rep. 6, 39166 (2016).

${ }^{11}$ F. Callewaert, S. Butun, Z. Li, and K. Aydin, Sci. Rep. 6, 32577 (2016).

${ }^{12}$ A. Ozer, N. Yilmaz, H. Kocer, and H. Kurt, J. Opt. 21, 055104 (2019).

${ }^{13}$ L. Zinkiewicz, J. Haberko, and P. Wasylczyk, Opt. Express 23, 4206-4211 (2015).

${ }^{14}$ P. Rodríguez-Ulibarri, V. Pacheco-Peña, M. Navarro-Cía, A. E. Serebryannikov, and M. Beruete, Appl. Phys. Lett. 106, 061109 (2015).

${ }^{15}$ H. Hajian, E. Ozbay, and H. Caglayan, Appl. Phys. Lett. 109, 031105 (2016).

${ }^{16}$ M. Beruete, A. E. Serebryannikov, V. Torres, M. Navarro-Cia, and M. Sorolla, Appl. Phys. Lett. 99, 154101 (2011).

${ }^{17}$ P. Rodriguez-Ulibarri, M. Beruete, M. Navarro-Cia, and A. E. Serebryannikov, Phys. Rev. B 88, 165137 (2013).

${ }^{18}$ T. Xu and H. J. Lezec, Nat. Commun. 5, 4141 (2014).

${ }^{19}$ C. L. Cortes, W. Newman, S. Molesky, and Z. Jacob, J. Opt. 14, 063001 (2012).

${ }^{20}$ A. K. Geim and K. S. Novoselov, Nat. Mater. 6, 183-191 (2007).
${ }^{\mathbf{2 1}}$ F. Bonaccorso, Z. Sun, T. Hasan, and A. C. Ferrari, Nat. Photonics 4, 611-622 (2010).

${ }^{22}$ A. N. Grigorenko, M. Polini, and K. S. Novoselov, Nat. Photonics 6, 749-758 (2012).

${ }^{23}$ L. A. Falkovsky, J. Phys. Conf. Ser. 129, 012004 (2008).

${ }^{24}$ Y. Zhou, Y. Dong, R. Fan, Q. Hu, R. Peng, and M. Wang, Appl. Phys. Lett. 105, 041114 (2014)

${ }^{25}$ Z. Li, W. Liu, H. Cheng, S. Chen, and J. Tian, Opt. Lett. 41, 3142-3145 (2016).

${ }^{26}$ J. Zhao, J. Zhang, Z. Zhu, X. Yuan, and S. Qin, J. Opt. 18, 095001 (2016).

${ }^{27}$ Y. Huang, Z. Yao, F. Hu, C. Liu, L. Yu, Y. Jin, and X. Xu, Carbon 119, 305-313 (2017).

${ }^{28}$ H. Jiang, W. Zhao, and Y. Jiang, Opt. Express 25, 19732-19739 (2017).

${ }^{29}$ T. Cao, Y. Li, X. Zhang, and Y. Zou, Photonics Res. 5, 441-449 (2017).

${ }^{30}$ I. Iorsh, I. S. Mukhin, I. V. Shadrivov, P. A. Belov, and Y. S. Kivshar, Phys, Rev. B 87, 075416 (2013).

${ }^{31}$ H. Hajian, A. Soltani-Vala, and M. Kalafi, Phys. Status Solidi C 9, 2614-2617 (2012).

${ }^{32}$ H. Hajian, H. Caglayan, and O. Ozbay, J. Appl. Phys. 121, 033101 (2017).

${ }^{33}$ Y.-C. Chang, C.-H. Liu, C.-H. Liu, S. Zhang, S. R. Marder, E. E. Narimanov, Z. Zhong, and T. B. Norris, Nat. Commun. 7, 10568 (2016).

${ }^{34}$ J. S. Gomez-Diaz, C. Moldovan, S. Capdevila, J. Romeu, L. S. Bernard, A. Magrez, A. M. Ionescu, and J. Perruisseau-Carrier, Nat. Commun. 6, 6334 (2015).

${ }^{35}$ A. Serebryannikov, H. Hajian, M. Beruete, E. Ozbay, and G. Vandenbosch, Opt. Mat. Express 8, 3887 (2018).

${ }^{36}$ A. Al Sayem, M. M. Rahman, M. R. C. Mahdy, I. Jahangir, and M. S. Rahman, Sci. Rep. 6, 25442 (2016).

${ }^{37}$ H. Hajian, A. Ghobadi, S. Abedini Dereshgi, B. Butun, and E. Ozbay, J. Opt. Soc. Am. B 34, D29-D35 (2017).

${ }^{38}$ J. D. Caldwell, A. V. Kretinin, Y. Chen, V. Giannini, M. M. Fogler, Y. Francescato, C. T. Ellis, J. G. Tischler, C. R. Woods, A. J. Giles, M. Hong, K. Watanabe, T. Taniguchi, S. A. Maier, and K. S. Novoselov, Nat. Commun. 5, 5221 (2014).

${ }^{39}$ S. Dai, Z. Fei, Q. Ma, A. S. Rodin, M. Wagner, A. S. McLeod, M. K. Liu, W. Gannett, W. Regan, K. Watanabe, T. Taniguchi, M. Thiemens, G. Dominguez, A. H. Castro Neto, A. Zettl, F. Keilmann, P. Jarillo-Herrero, M. M. Fogler, and D. N. Basov, Science 343, 1125 (2014).

${ }^{40} \mathrm{H}$. Hajian, A. Ghobadi, B. Butun, and E. Ozbay, Opt. Express 26, 16940 (2018).

${ }^{41}$ H. Hajian, A. Ghobadi, A. Serebryannikov, B. Butun, G. Vandenbosch, and E. Ozbay, J. Opt. Soc. Am. B 36, 1607 (2019).

${ }^{\mathbf{4 2}}$ See http://www.lumerical.com/tcad-products/fdtd/for Lumerical Solutions, Inc. ${ }^{43}$ S. Butun and K. Aydin, ACS Photonics 2, 1652-1657 (2015).

${ }^{44}$ A. E. Serebryannikov, S. Nojima, and E. Ozbay, Phys. Rev. B 90, 235126 (2014).

${ }^{45}$ P. Rodríguez-Ulibarri, M. Beruete, and A. E. Serebryannikov, Phys. Rev. B 96, 155148 (2017)

${ }^{46}$ H. Hajian, A. Ghobadi, B. Butun, and E. Ozbay, J. Opt. Soc. Am. B 36, F131 (2019). 